\title{
Incidence of malignancy and survival in patients with idiopathic pleuritis
}

\author{
Simon Bertram Reuter ${ }^{1,2}$, Paul Frost Clementsen ${ }^{3,4,5,6}$, Uffe Bodtger ${ }^{1,2,3}$ \\ ${ }^{1}$ Department of Respiratory Medicine, Naestved Hospital, Naestved, Denmark; ${ }^{2}$ Institute of Regional Health Research, University of Southern \\ Denmark, Odense C, Denmark; ${ }^{3}$ Department of Internal Medicine, Zealand University Hospital, Roskilde, Roskilde, Denmark; ${ }^{4}$ Copenhagen \\ Academy for Medical Education and Simulation (CAMES), Rigshospitalet, Copenhagen, Denmark; ${ }^{5}$ University of Copenhagen, Copenhagen, \\ Denmark; ${ }^{6}$ Department of Clinical Medicine, University of Copenhagen, Copenhagen, Denmark \\ Contributions: (I) Conception and design: SB Reuter, U Bodtger; (II) Administrative support: SB Reuter, U Bodtger; (III) Provision of study materials \\ or patients: SB Reuter; (IV) Collection and assembly of data: SB Reuter; (V) Data analysis and interpretation: All authors; (VI) Manuscript writing: \\ All authors; (VII) Final approval of manuscript: All authors. \\ Correspondence to: Simon Bertram Reuter. 61, Ringstedgade, DK-4700 Naestved, Denmark. Email: simonreuter@dadlnet.dk.
}

Background: The long-term outcome after non-diagnostic thoracoscopy (idiopathic pleuritis) has not been investigated in nationwide studies, and the survival has never been estimated. Therefore, we decided to investigate the three-year incidence of malignancy and survival of patients with idiopathic pleuritis.

Methods: Retrospective, register-based, nationwide study of patients undergoing diagnostic video-assisted thoracoscopic surgery (VATS) thoracoscopy $\leq 30$ days after thoracentesis, using The National Patient Registry and The Danish Cancer Registry. Idiopathic pleuritis was defined as; no diagnosis of malignancy within 31 days after VATS. Patients were followed for 36 months after VATS.

Results: In total, idiopathic pleuritis were identified in 547 out of 658 patients undergoing VATS (83\%), and 29 (5\%) were diagnosed with malignancy during the 3 years follow-up period after VATS. Of these, 93\% were diagnosed with malignancy within the first year. Numbers-needed-to-follow-up for detecting one case of malignancy was 18 during the first year after VATS and 250 in the two subsequent years. Survival was independent on type of malignancy (MPM vs. other malignancies; $\mathrm{P}=0.13$ ) and of time from VATS to diagnosis ( $\leq 31$ days vs. 1-36 months; $\mathrm{P}=0.15$ ). Median survival in the non-malignant group was 1,095 days.

Conclusions: Our study confirms a low incidence of malignancy in idiopathic pleuritis after VATS. Nearly all incident cases of malignancy were diagnosed within 12 months from VATS. No survival disadvantage was observed in patients with incident malignancy. Our data suggest that follow-up of idiopathic pleuritis could safely be limited to 1 year. The optimal follow-up strategy remains to be investigated.

Keywords: Thoracoscopy; thoracic surgery; video-assisted; pleurisy; pleural effusion

Submitted Sep 24, 2018. Accepted for publication Dec 17, 2018.

doi: $10.21037 /$ jtd.2018.12.136

View this article at: http://dx.doi.org/10.21037/jtd.2018.12.136

\section{Introduction}

In approximately $25 \%$ of patients with a unilateral pleural effusion, a thoracoscopic pleural biopsy shows no histological findings suggesting a possible etiology (1-8). Such cases are labelled non-specific pleuritis or idiopathic pleuritis, an umbrella term for a wide range of conditions including malignant pleural effusions (3-5).
Seven retrospective, long-term follow-up studies, including from 44 to 391 patients have been published regarding idiopathic pleuritis after thoracoscopy performed as either pleuroscopy $(2-5,8)$ or video-assisted thoracoscopic surgery (VATS) $(6,7)$. The majority of the studies followed the patients for 2 years, yet with considerable variation: DePew et al. 7 (range, 2-10) months (6), Gunluoglu et al. 24 (range, 6-60) months (7), Venekamp et al. 33 (range, 5- 
Table 1 Baseline characteristics of included patients $(n=872)$

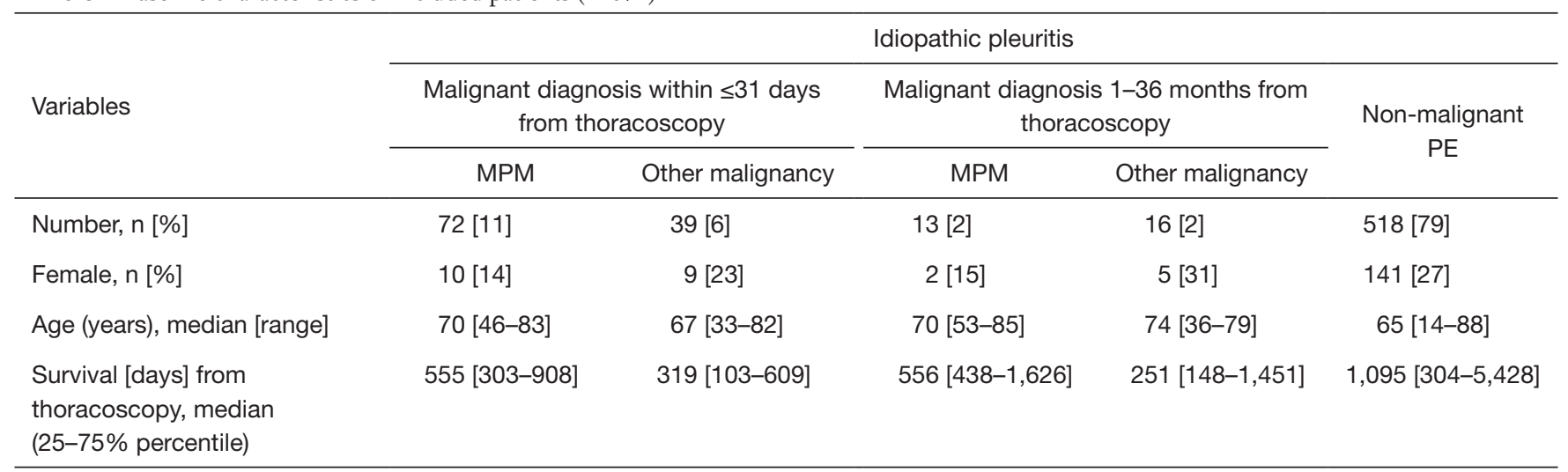

60) months (3), and Yang et al. 35 (range, 1-143) months (8). Pleural malignancy was diagnosed during follow-up in $3.5-18 \%$ of cases, dominated by malignant pleural mesothelioma (MPM) (2-8).

All studies were single-center studies from highly specialized centers, no multicenter studies on neither incidence of malignancy nor mortality in idiopathic pleuritis after thoracoscopy exist.

On this background, we decided to perform a nationwide study investigating the incidence of malignancy and survival of patients with idiopathic pleuritis, i.e., unresolved pleural effusions after VATS.

\section{Methods}

\section{Design}

Retrospective, observational, register-based, nationwide study including three years of follow-up in all patient undergoing a diagnostic VATS thoracoscopy $\leq 30$ days after thoracentesis. The study was registered at clinicaltrials.gov (NCT03270228).

\section{Registries}

All Danish citizens are at birth provided a unique and permanent personal 10-digit identification code (the Danish Civil Registration Number), which is used in all national registries (9). The National Patient Registry has kept records of all hospital visits (out-patient or admissions) since 1978. Each hospitalization is coded according to the International Classification of Diseases $8^{\text {th }}$ revision (until 1994) and $10^{\text {th }}$ revision (from 1994) (10). The Danish Cancer Registry holds records of all incidences of malignant neoplasms including tumor characteristics from 1943 (11). Vital status was available from the civil registration system (12). We linked information from the above registries for each patient, using the Danish Civil Registration Number.

\section{Inclusion and exclusion criteria}

All patients in the National Patient Registry who underwent a VATS procedure between January 1996 and December 2013 AND preceded by a non-diagnostic thoracentesis $\leq 30$ days.

If a patient had more than one VATS performed, the first was used as index for the analysis. We excluded patients with known malignancy at the time of index VATS.

\section{Outcomes}

The primary outcome was the three-year incidence of malignancy in patients with idiopathic pleuritis. Secondary outcomes were survival including comparison between patients diagnosed with a malignancy $\leq 31$ days versus $1-36$ months after VATS.

\section{Definition}

We defined a malignancy associated pleural effusion as patients who were registered with a malignant diagnosis $\leq 31$ days from VATS. We defined an idiopathic pleural effusion as patients with no malignancy diagnosed $\leq 31$ days from VATS, see Table 1.

Based on the above, patients with idiopathic pleuritis consisted after three years of two groups: those who were respectively were not diagnosed with malignancy 


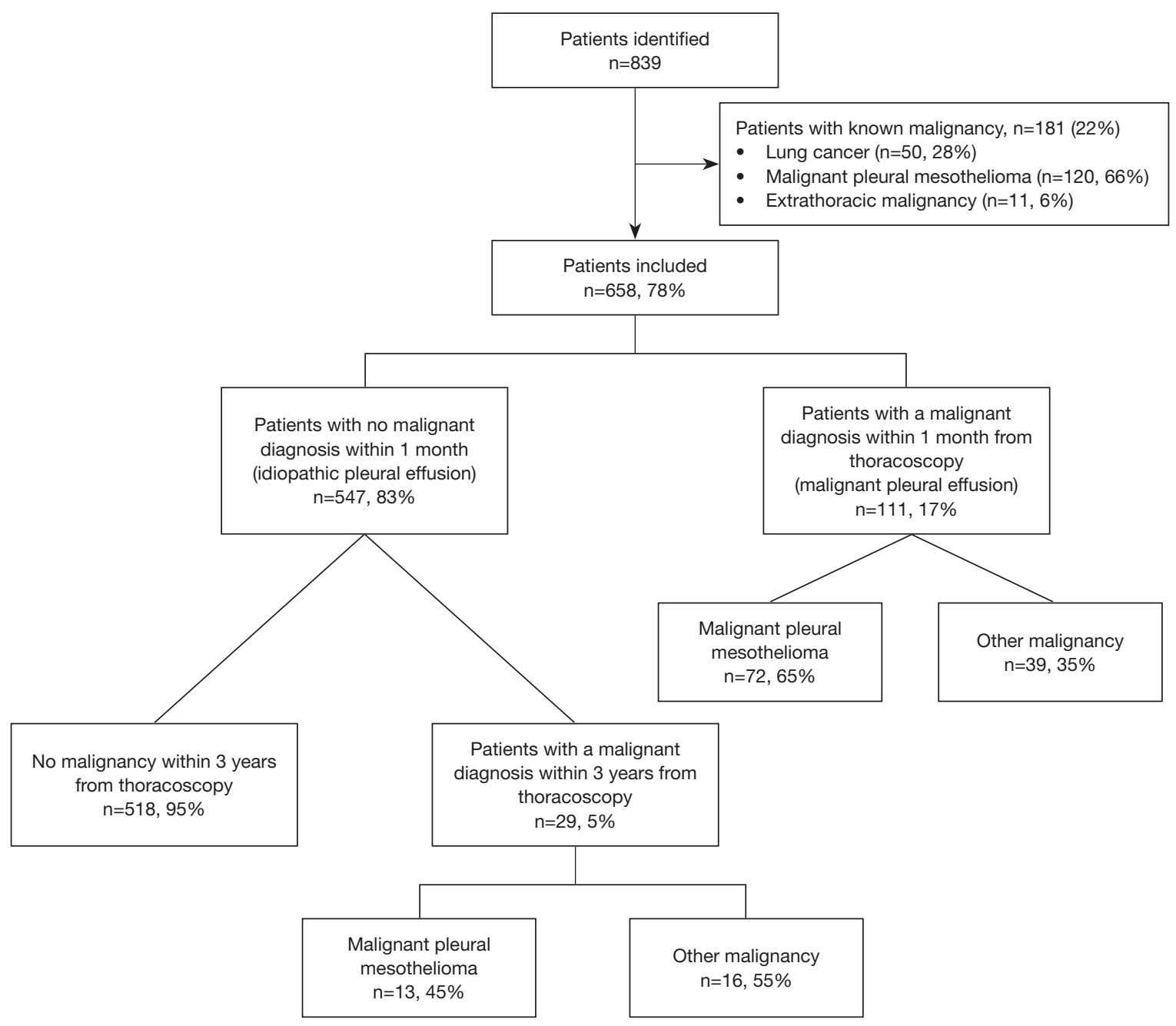

Figure 1 Overview of the study population.

(1-36 months from VATS), see Table 1.

\section{Statistics}

Data were presented as number and frequencies, median and range or $25^{\text {th }}$ and $75^{\text {th }}$ percentiles. The chi-squared test was used for comparing continuous data. The survival was depicted using Kaplan-Meier survival curves, and Log-rank test was used for comparing groups. We adjusted for age and gender, and because the clinical work-up has changed during the last 20 years including the general prognosis for cancer, we in addition adjusted for the year of thoracoscopy. Patients who died during follow-up was censored in the survival analysis. Data were analyzed using STATA (StataCorp LLC, Version 15.0, College Station, Texas, USA).

\section{Ethics}

Retrospective register studies do not require ethical approval from the authorities in Denmark. The study was approved by the Danish Data Protection Agency (REG-872016). Data were anonymized.

\section{Results}

We identified 839 patients and 658 patients were included in the analysis, see Figure 1 and Table 1.

\section{Incidence of malignancy in patients with idiopathic} pleuritis during follow-up (1 to 36 months after VATS)

One month after VATS, 547 patients (83\%) were not 
Table 2 Comparison of types of cancer between patients with malignancy diagnosed within 1 month from VATS thoracoscopy $v s$. patients with malignancy following an initial diagnosis of idiopathic pleuritis.

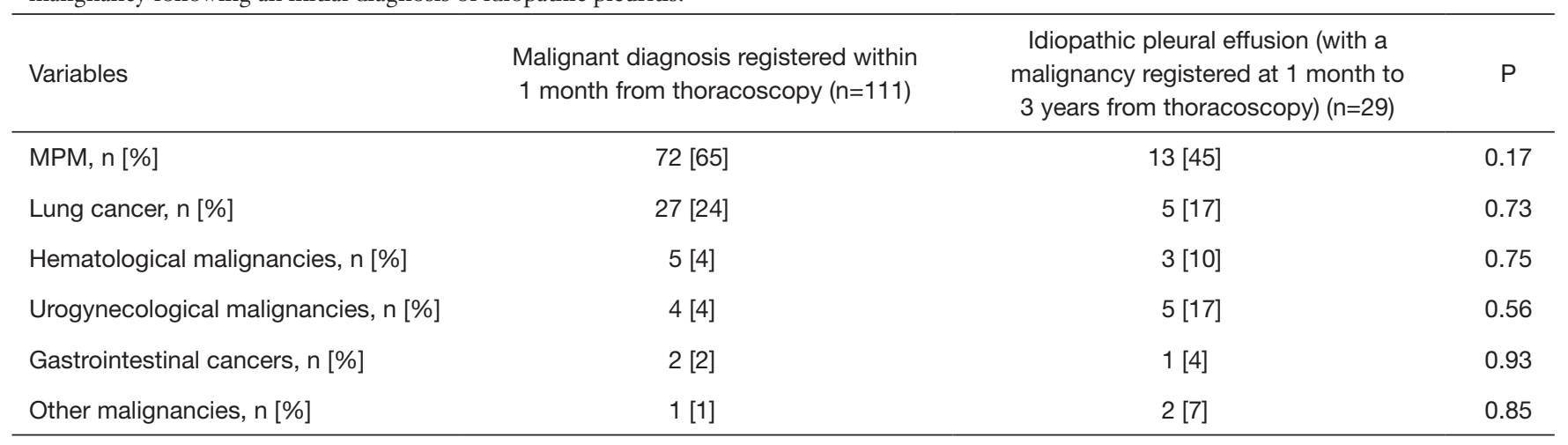

VATS, video-assisted thoracoscopic surgery; MPM, malignant pleural mesothelioma.

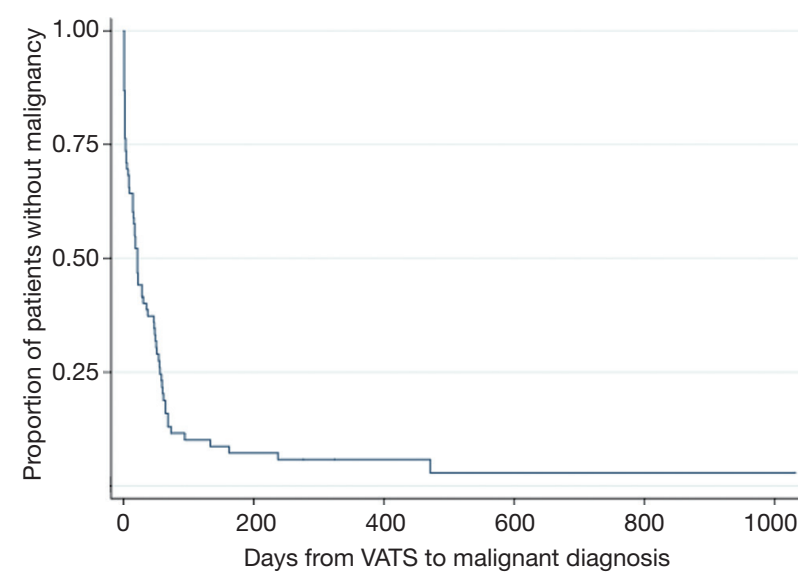

Figure 2 Time from thoracoscopy to malignancy diagnosed in patients with idiopathic malignant pleural effusions. VATS, videoassisted thoracoscopic surgery.

diagnosed with malignancy and were classified as having idiopathic pleuritis.

Of these, 29 patients ( $5 \%$ ) were diagnosed with malignancy during the 3 -year follow-up period after VATS, see Figure 1 and Tables 1 and 2.

Figure 2 shows that the majority of these patients were diagnosed with malignancy within a limited time span: $15 / 29$ patients $(52 \%)$ were diagnosed within the first two months, 24/29 (83\%) during the first 6 months and 27/29 patients $(93 \%)$ within the first year after VATS. The numbers-needed-to-follow up during the first year was 18 to identify one case of malignancy.

Totally, 520 patients were not diagnosed with malignancy 1 year after VATS (Figure 2, Table 1) but one patient was diagnosed at 471 days (malignant pleural mesothelioma) and one at 1,031 days (diffuse large B-cell lymphoma) after VATS. Thus, the incidence of malignancy was $0.4 \%(2 / 520)$ during the period of 1-3 years after VATS, corresponding to a numbers-needed-to-follow-up of 260 patients to identify one case of malignancy. Concerning the time period from 6 months to 3 years after VATS, the incidence of malignancy was $1.3 \%(7 / 525)$ corresponding to a numbers-needed-tofollow-up of 75 patients to identify one case of malignancy.

\section{Incidence of malignancy during initial workup ( $\leq 31$ days after VATS)}

Of the included patients, 111 (17\%) were diagnosed with a malignancy within 31 days from VATS, see Figure 1 and Tables 1 and 2. Malignant pleural mesothelioma ( $\mathrm{n}=72 ; 65 \%)$ and lung cancer $(n=27 ; 24 \%)$ were the major causes, see Table 2 .

\section{Difference in type of malignancy diagnosed early $(\leq 31$ days after VATS) versus late (1 to 36 months after VATS)}

Table 2 depicts the types of malignancy in patients diagnosed $\leq 31$ days $v s$. 1-36 months after VATS. There was no difference in the incidence of malignancy comparing patients diagnosed early $v s$. late.

\section{Survival}

Figure 3 depicts the survival curves and Table 3 the corresponding numbers. Table 1 shows the median days of survival after VATS. Overall, patients diagnosed with malignancy lived significantly shorter after VATS than patients with no malignant diagnoses $(\mathrm{P}<0.001)$, however 
the median survival was around three years (Table 1).

Survival was independent on type of malignancy (MPM $v s$. other malignancies; $\mathrm{P}=0.13$ ) and of time from VATS to diagnosis ( $\leq 31$ days $v s$. $1-36$ months; $\mathrm{P}=0.15)$.

\section{Discussion}

In the current study on patients with idiopathic pleuritis after VATS, we found an incidence of malignancy of $5 \%$. Median survival from the initial VATS did not differ significantly between patients diagnosed early ( $\leq 31$ days from VATS) $v s$. during follow-up (1-36 months after VATS).

Our findings were equal to cancer incidences found in four of the seven previous single-center studies (3.5-8.3\%) $(2,3,6,7)$. Several aspects might explain the disagreement with the three remaining studies. Most importantly, they all used pleuroscopy and not VATS as reference standard.

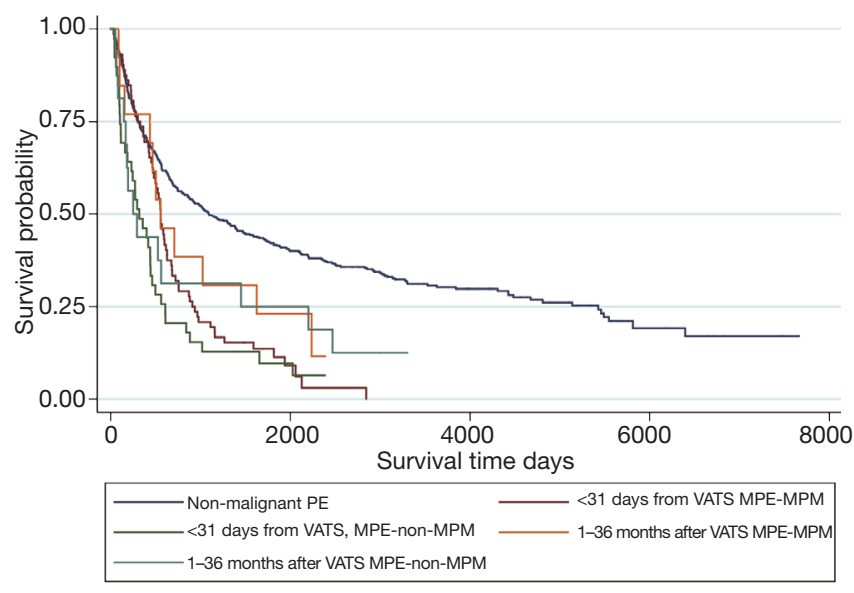

Figure 3 Kaplan-Meier plot of the five patient groups. VATS, video-assisted thoracoscopic surgery.
Additionally, differences in levels of asbestos exposure might have additional significance: One study from England performed in a tertiary center with an unreported but "high incidence of MPM", found an incidence of malignancy of $12 \%$ after follow-up (4). One study from Turkey performed in an area with 60-80 times higher prevalence of MPM compared to Denmark (5), reported an incidence malignancy of $18 \%$. The last study, from China, found an incidence of malignancy of $15 \%$ (8). The reported prevalence of MPM in China is lower than would be expected based on asbestos use and MPM-related mortality (third highest in the world) (13). However, another study from Turkey, using VATS as standard reference, found a comparable incidence of malignancy in idiopathic pleuritis as in our study (7). This may suggest that VATS have superior sensitivity to pleuroscopy in ruling out malignancy in pleural effusions of unknown origin $(7,14)$. However, inter-center differences in experience of thoracoscopists and/or pathologists might also be of importance (15). No prospective studies so far have compared the sensitivity of pleuroscopy and VATS. A systematic review and meta-analysis found that pleuroscopy using a semirigid thoracoscope had a sensitivity of $91 \%$ and a specificity of $100 \%$ (including both malignant and nonmalignant diagnoses) in 755 patients with pleural exudates (17 studies) (16). However, data for true negative cases were only available in seven studies, and only two studies provided data about follow-up duration (16).

Additionally, we found that most patients were diagnosed with malignancy during the first months after index VATS. Previously, four studies found that the majority of patients were diagnosed within one year after thoracoscopy $(2,4,6,8)$, whereas the remaining studies did not state time from thoracoscopy to diagnosis $(3,5,7)$.

Our data shows a low incidence $(1.3 \%)$ of malignancy after

Table 3 Cumulated number of patients who died during follow-up (n, \%)

\begin{tabular}{|c|c|c|c|c|c|c|}
\hline Variables & 31 days & 3 months & 6 months & 1 year & 2 years & 3 years \\
\hline \multicolumn{7}{|c|}{ Diagnosed during initial workup } \\
\hline MPM $(n=72)$ & 0 & $4(6 \%)$ & $9(13 \%)$ & $19(26 \%)$ & $48(67 \%)$ & $56(78 \%)$ \\
\hline Other malignancy $(n=39)$ & 0 & $5(13 \%)$ & $12(31 \%)$ & $20(51 \%)$ & $30(77 \%)$ & $34(87 \%)$ \\
\hline \multicolumn{7}{|l|}{ Idiopathic pleuritis group } \\
\hline Other malignancy $(n=16)$ & 0 & $3(19 \%)$ & $5(31 \%)$ & $8(50 \%)$ & $10(63 \%)$ & $10(64 \%)$ \\
\hline No malignancy $(n=518)$ & 0 & $29(6 \%)$ & 77 (15\%) & $149(29 \%)$ & $222(43 \%)$ & 259 (50\%) \\
\hline
\end{tabular}

MPM, malignant pleural mesothelioma. 
completing the first 6 months of follow-up after index VATS.

On the other hand, numbers needed to follow-up for identifying one case of malignancy during the first 6 months was 18 . This might influence future decisions on followup intensity and duration of idiopathic pleuritis. The lower the pre-test probability, the higher the risk of false-positive results, thus we have concerns about potentially harming the vast majority (here: $98.7 \%$ ) without malignancy.

Median survival in patients with idiopathic pleuritis without a diagnosis of malignancy during follow-up was surprisingly low (3 years; Table 1). This may indicate that being evaluated for possible malignancy-associated pleural effusion by VATS, i.e., having recurrent pleural effusion is a marker of significant and serious yet non-malignant condition. It is well-known that pleural effusions may in many cases have more than one cause (17). However, there are no long-term prospective studies on possible benefits or risks of follow-up in these patients, yet systematic evaluation for thyroid, renal, hepatic and heart disease increased the number of targetable diagnoses (18). One could also consider under-diagnosis of malignancy as Table 3 shows similar mortality rates in all groups during follow-up. The current paper does not address cause of mortality, which could be an issue of futures studies. A recent US autopsy study indicates that undiagnosed malignancy related to cause of death is rare (3.1\% of cases) (19).

To the best of our knowledge, this is the largest $(>600$ patients) and first nationwide study with a uniform followup (3-years) of patients with idiopathic pleuritis. The Danish health care system is free for all Danish citizens, and there are no private hospitals or services performing any kind of thoracoscopy or other pleural procedures. The unique personal registration number allows fusion of several national databases, allowing high data completion and includes diagnoses from out-patient clinics, admission and emergency-care.

However, the registry-based design has several limitations. Firstly, our study concerns predominantly VATS, not pleuroscopy. In Denmark, workup of pleural effusions is performed in 14 pulmonology centers: 12 centers refer to VATS at the local department of thoracic surgery if thoracoscopy is needed. Only two centers offer pleuroscopy (20). Thus, the majority of thoracoscopies in Denmark are performed as VATS procedures.

Secondly, we arbitrarily chose a maximum time span from thoracentesis to thoracoscopy of $\leq 30$ days. Increasing this time span, we would have identified additional patients, yet also introduced heterogeneity in our study population (e.g., patients undergoing VATS for other reasons than a pleural effusion). Therefore, there is a risk that we might have underestimated the number of patients with idiopathic pleural effusions and hence malignancy.

Thirdly, the categorization of receiving a diagnosis within or later than 1 month after VATS is arbitrary. However, in Denmark histopathological analyses are finished within 10 days, often shorter. The Danish National Patient Registry includes diagnoses from all kind of hospital contacts, thus the time spent from final histopathological analysis to patient information and diagnosis registry is much less than 31 days. Thus, we consider 1 month after VATS to be sufficient time to allow for both biopsy handling, lab analyses, patient information and diagnosis registration.

Fourthly, tuberculosis is a common cause worldwide of idiopathic pleuritis. However, in Denmark, the incidence of pleural tuberculosis is very low (2016: 11 cases $=2.0 \mathrm{pr}$. million inhabitants) (21). The external validity regarding risk of incidence of malignancy in idiopathic pleuritis might therefore be limited to countries with similar rates of TB as well as asbestos exposure.

Lastly, as discussed earlier a substantial number of patients died during follow-up in all groups, see Table 3. Thus, it is possible that patients classified as nonmalignant idiopathic pleuritis may have died from (or with) undiagnosed malignancy. Future studies will clarify the likelihood of missed malignant diagnoses.

Our study do not provide insight into which procedure that provided the histopathological evidence of malignancy, neither in those diagnosed early nor in those diagnosed during follow-up. Our study shows that the risk in the idiopathic pleuritis group of being diagnosed with malignancy decreased rapidly within the first year after VATS: numbers-needed-to-follow-up increased to 260 after the first year. In summary, we suggest that routine follow-up of pleuritis after inconclusive VATS thoracoscopy is limited to 1 year $(6,8)$, and follow-up includes medical history, clinical examination and chest ultrasound after 2, 6, and 12 months. This initial status guides the use of further testing such as other imaging (CT, PET-CT) and/or invasive workup. Future clinical and prospective studies are needed to clarify the ideal follow-up program.

\section{Conclusions}

This is the largest follow-up study on idiopathic pleuritis, and the only reporting national data and using uniform follow-up time (3 years). Overall, our study confirms a low incidence of malignancy in idiopathic pleuritis after VATS 
thoracoscopy (5\%), with $93 \%$ being diagnosed within 1 year after VATS.

We suggest a schedule for follow-up of idiopathic pleuritis limited to 12 months and limiting routine use of CT. Our study needs repetition in countries with higher incidences of pleural tuberculosis.

\section{Acknowledgements}

None.

\section{Footnote}

Conflicts of Interest: The authors have no conflicts of interest to declare.

Ethical Statement: Retrospective register studies do not require ethical approval from the authorities in Denmark. The study was approved by the Danish Data Protection Agency (REG-87-2016). Data were anonymized.

\section{References}

1. Wrightson JM, Davies HE. Outcome of patients with nonspecific pleuritis at thoracoscopy. Curr Opin Pulm Med 2011;17:242-6.

2. Janssen JP, Ramlal S, Mravunac M. The LongTerm Follow Up of Exudative Pleural Effusion After Nondiagnostic Thoracoscopy. Journal of Bronchology \& Interventional Pulmonology 2004;11:169-74.

3. Venekamp LN, Velkeniers B, Noppen M. Does 'idiopathic pleuritis' exist? Natural history of non-specific pleuritis diagnosed after thoracoscopy. Respiration 2005;72:74-8.

4. Davies HE, Nicholson JE, Rahman NM, et al. Outcome of patients with nonspecific pleuritis/fibrosis on thoracoscopic pleural biopsies. Eur J Cardiothorac Surg 2010;38:472-7.

5. Metintas M, Ak G, Cadirci O, et al. Outcome of patients diagnosed with fibrinous pleuritis after medical thoracoscopy. Respir Med 2012;106:1177-83.

6. DePew ZS, Verma A, Wigle D, et al. Nonspecific pleuritis: optimal duration of follow-up. Ann Thorac Surg 2014;97:1867-71.

7. Gunluoglu G, Olcmen A, Gunluoglu MZ, et al. Longterm Outcome of Patients With Undiagnosed Pleural Effusion. Arch Bronconeumol 2015;51:632-6.

8. Yang $\mathrm{Y}, \mathrm{Wu} \mathrm{YB}$, Wang $\mathrm{Z}$, et al. Long-term outcome of patients with nonspecific pleurisy at medical thoracoscopy. Respir Med 2017;124:1-5.
9. Thygesen LC, Daasnes C, Thaulow I, et al. Introduction to Danish (nationwide) registers on health and social issues: structure, access, legislation, and archiving. Scand J Public Health 2011;39:12-6.

10. Schmidt M, Schmidt SA, Sandegaard JL, et al. The Danish National Patient Registry: a review of content, data quality, and research potential. Clin Epidemiol 2015;7:449-90.

11. Gjerstorff ML. The Danish Cancer Registry. Scand J Public Health 2011;39:42-5.

12. Schmidt M, Pedersen L, Sorensen HT. The Danish Civil Registration System as a tool in epidemiology. Eur J Epidemiol 2014;29:541-9.

13. Park EK, Takahashi K, Hoshuyama T, et al. Global magnitude of reported and unreported mesothelioma. Environ Health Perspect 2011;119:514-8.

14. Baris YI, Grandjean P. Prospective study of mesothelioma mortality in Turkish villages with exposure to fibrous zeolite. J Natl Cancer Inst 2006;98:414-7.

15. Guo Z, Carbone $M$, Zhang $X$, et al. Improving the Accuracy of Mesothelioma Diagnosis in China. J Thorac Oncol 2017;12:714-23.

16. Agarwal R, Aggarwal AN, Gupta D. Diagnostic accuracy and safety of semirigid thoracoscopy in exudative pleural effusions: a meta-analysis. Chest 2013;144:1857-67.

17. Bintcliffe OJ, Hooper CE, Rider IJ, et al. Unilateral Pleural Effusions with More Than One Apparent Etiology. A Prospective Observational Study. Ann Am Thorac Soc 2016;13:1050-6.

18. Walker SP, Morley AJ, Stadon L, et al. Nonmalignant Pleural Effusions: A Prospective Study of 356 Consecutive Unselected Patients. Chest 2017;151:1099-105.

19. Parajuli S, Aneja A, Mukherjee A. Undiagnosed fatal malignancy in adult autopsies: a 10-year retrospective study. Hum Pathol 2016;48:32-6.

20. Willendrup F, Bodtger U, Colella S, et al. Diagnostic accuracy and safety of semirigid thoracoscopy in exudative pleural effusions in Denmark. J Bronchology Interv Pulmonol 2014;21:215-9.

21. Fally M, Reuter S, Ambruster K, et al. Pleural effusion - Investigation and Treatment. The Danish Society of Respiratory Medicine, Guideline 2017. Available online: https://www.lungemedicin.dk/fagligt/69-pleuraeffusion.html

Cite this article as: Reuter SB, Clementsen PF, Bodtger U. Incidence of malignancy and survival in patients with idiopathic pleuritis. J Thorac Dis 2019;11(2):386-392. doi: 10.21037/ jtd.2018.12.136 